\title{
Massive online open course como estratégia para o ensino de segurança no processo de medicação
}

\author{
Massive online open course as a strategy for teaching medication safety
}

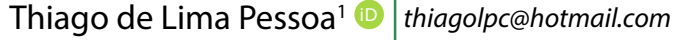 \\ Zenewton André da Silva Gama' (D) zasgama@gmail.com \\ Paulo José de Medeiros' (1) paulojmedeiros1967@gmail.com \\ Marise Reis de Freitas' ${ }^{1}$ (D) mariserf@gmail.com
}

\begin{abstract}
RESUMO
Introdução: Erros de medicação são comuns e causam sofrimento e custos que podem ser evitados. A mitigação da ocorrência de eventos adversos a medicamentos é tratada como prioridade pela Organização Mundial da Saúde (OMS), que recomenda a inclusão do tema segurança do paciente nos currículos das profissões de saúde como medida para minimizar danos aos pacientes. Um curso on-line aberto e massivo (MOOC) sobre segurança no processo de medicação foi desenvolvido como estratégia educacional para a área da saúde e disponibilizado no Ambiente Virtual de Aprendizagem do Sistema Único de Saúde (Avasus), em março de 2018.
\end{abstract}

Objetivo: Este estudo teve como objetivo avaliar a satisfação e o aprendizado dos participantes que concluíram o curso.

Método: Trata-se de estudo de delineamento quase experimental não controlado. O MOOC possui uma carga horária de 30 horas e aborda temas sobre segurança na prescrição, dispensação, administração e notificação de incidentes relacionados a medicamentos. Aplicaram-se um préteste e um pós-teste de múltipla escolha para avaliar o nível de conhecimento dos participantes, e um questionário de satisfação foi respondido ao término do curso. Analisaram-se a confiabilidade dos instrumentos de medida, a significância da melhoria do conhecimento e os fatores associados (sexo, idade e profissão).

Resultados: Dos 7.135 participantes inscritos no período de março a dezembro de 2018, $2.902(40,7 \%)$ responderam aos instrumentos de pesquisa. A confiabilidade do questionário de satisfação foi boa (alfa de Cronbach =0,83), e o curso foi bem avaliado como estratégia educacional $(96,1 \%)$. Em relação ao nível de conhecimento, observou-se aumento significativo (antes $=8,10$; depois $=8,74 ; p<0,05$ ) com melhoria relativa de $32 \%$. O fator idade esteve diretamente associado à aprendizagem ( $\beta=1,36 ; \mathrm{IC} 95 \%=0,35-2,36 ; p=0,008)$.

Conclusão: A utilização do MOOC mostrou-se efetiva para o ensino de segurança no processo de medicação, ferramenta que pode ser utilizada para a inserção do tema segurança do paciente nos currículos das profissões de saúde.

Palavras-chave: Segurança do Paciente; Educação Continuada; Educação a Distância; Erros de Medicação.

\section{ABSTRACT}

Introduction: Medication errors are common and cause suffering and costs that can be prevented. The mitigation of adverse drug events is treated as a priority by the World Health Organization (WHO), which recommends including the subject of patient safety in the curricula of health profession courses to minimize harm to patients. An open and massive online course (MOOC) on safety in the medication process was developed as an educational strategy for the health area, which was made available through the SUS Virtual Learning Environment (AVASUS) in March 2018.

Objective: To estimate the satisfaction and learning of the participants who finished the course.

Method: Uncontrolled quasi-experimental design study. The MOOC consists of 30 hours of learning and addresses topics on safety in prescription, dispensing, administration and notification of incidents related to medications. Multiple choice questions were applied pre-and post-test to assess the participants' level of knowledge and a satisfaction survey was answered at the end of the course. The reliability of the measurement instruments, the significance of improved knowledge and associated factors (sex, age and profession) were analyzed.

Results: Of the 7,135 registered participants, from March to December 2018, 2,902 (40.7\%) responded to the survey instruments. The reliability of the satisfaction questionnaire was good (Cronbach's alpha $=0.83$ ) and the course was well evaluated as an educational strategy $(96.1 \%)$. Regarding the level of knowledge, a significant increase was observed (before $=8.10$; after $=8.74 ; p<0.05$ ) with a relative improvement of $32 \%$. The age factor was directly associated with learning $(\beta=1.36 ; 95 \% \mathrm{Cl}=0.35-2.36 ; p=0.008)$.

Conclusion: The use of $M O O C$ proved to be effective for teaching patient safety in the medication process, a tool that can be used to insert this subject in the curricula of health profession courses.

Keywords: Patient Safety; Continuing Education; E-learning; Medication Errors.

${ }^{1}$ Universidade Federal do Rio Grande do Norte, Natal, Rio Grande do Norte, Brasil.

Editora-chefe: Daniela Chiesa

Editora associada: Izabel Cristina Meister Martins Coelho

Recebido em 14/11/19; Aceito em 18/01/21.

Avaliado pelo processo de double blind review. 


\section{INTRODUÇÃO}

A Organização Mundial da Saúde (OMS) lançou o terceiro Desafio Global "Uso seguro de medicamentos" ("Medication without harm"), o qual elenca soluções para a garantia da segurança no processo de medicação, com o objetivo de reduzir $50 \%$ dos danos evitáveis relacionados a medicamentos em um prazo de cinco anos ${ }^{1}$. Eventos adversos a medicamentos (EAM), além de outros eventos, aumentam o tempo de internação e os custos hospitalares, assim como trazem consequências mais sérias, como sequelas e óbitos ${ }^{2-6}$. Os EAM causam ao menos uma morte todos os dias e prejudicam aproximadamente 1,3 milhão de pessoas anualmente, apenas nos Estados Unidos. No mundo, o custo associado aos eventos preveníveis relacionados a medicamentos foi estimado em US\$ 42 bilhões por ano, representando quase $1 \%$ do total das despesas com saúde1.

Uma das estratégias globais para o enfrentamento desse problema é a ênfase em formação dos profissionais de saúde em segurança do paciente. Desde 2008, a OMS tem liderado a proposição de guias curriculares para induzir e qualificar o ensino dessa temática na área da saúde, de forma que estudantes e profissionais estejam bem informados e capacitados quanto aos princípios e às práticas seguras na assistência aos pacientes ${ }^{7}$.

No Brasil, o ensino de segurança do paciente tem sido promovido por políticas públicas de saúde, como o Programa Nacional de Segurança do Paciente (PNSP). Esse programa foi um marco para a implementação da cultura de segurança do paciente no país, tendo como objetivo geral contribuir para a qualificação do cuidado em saúde em todos os estabelecimentos de saúde do território nacional, com a criação dos Núcleos de Segurança do Paciente (NSP). Um dos eixos estruturantes do PNSP é a inclusão do tema segurança do paciente no ensino técnico, na graduação e pós-graduação na área da saúde, incluindo os programas de residência em saúde, e em processos de educação permanente ${ }^{8,9}$.

Nessecontexto, aeducaçãoa distância(EaD)surgecomouma alternativa para a difusão das práticas e para o ensino de segurança do paciente, pois permite ao aluno a compatibilização do curso com suas possibilidades de horário, a realização no ritmo desejado e em qualquer espaço disponível, além do desenvolvimento de habilidades e atitudes, como independência, comportamento proativo e autodisciplina na busca de seu desenvolvimento. Para isso, o ambiente virtual de aprendizagem (AVA) torna-se um meio propício para o ensino e a capacitação de alunos e profissionais de saúde. Assim, a EaD representa uma modalidade factível nos cenários de prática e é considerada uma importante contribuição para novas oportunidades de aprendizagem ${ }^{10}$.

Diante da necessidade de formação de profissionais em segurança do paciente em larga escala, desenvolveu- se um componente curricular sobre segurança no processo de medicação utilizando um curso on-line aberto e massivo (massive open online course - MOOC), que é um recurso potencialmente efetivo para essa demanda. O objetivo desta publicação é avaliar a satisfação e o aprendizado dos participantes que concluíram o curso.

\section{MÉTODO}

Trata-se de um estudo de delineamento quase experimental não controlado com o desenvolvimento e a avaliação de um MOOC. O módulo educacional foi desenvolvido sob a orientação e a coordenação da Secretaria de Educação a Distância da Universidade Federal do Rio Grande do Norte (UFRN), no período de janeiro de 2017 a fevereiro de 2018.

\section{Oferta educacional}

O módulo educacional com carga horária de 30 horas foi escrito utilizando-se uma linguagem dialogada e interativa. Histórias em quadrinhos e um jogo virtual problematizam situações relacionadas ao processo de medicação de maneira lúdica e interativa. O curso está organizado em quatro unidades (Quadro 1) dispostas na plataforma em textos em formato PDF.

Quadro 1.Conteúdo programático do módulo

\begin{tabular}{|c|c|c|}
\hline Unidade & Aula & Título \\
\hline \multirow{4}{*}{$\begin{array}{l}\text { Unidade } 1: \text { A } \\
\text { importância da } \\
\text { prescrição segura } \\
\text { de medicamentos. }\end{array}$} & Aula 1 & $\begin{array}{l}\text { Por que falar em segurança } \\
\text { do paciente? }\end{array}$ \\
\hline & Aula 2 & $\begin{array}{l}\text { Como prescrever de forma } \\
\text { segura? }\end{array}$ \\
\hline & Aula 3 & $\begin{array}{l}\text { Cuidados nas prescrições de } \\
\text { medicamentos injetáveis }\end{array}$ \\
\hline & Aula 4 & $\begin{array}{l}\text { O que mais pode ser evitado } \\
\text { nas prescrições? }\end{array}$ \\
\hline \multirow{3}{*}{$\begin{array}{l}\text { Unidade 2: } \\
\text { Compreendendo } \\
\text { a dispensação } \\
\text { segura de } \\
\text { medicamentos. }\end{array}$} & Aula 1 & $\begin{array}{l}\text { Entendendo os sistemas } \\
\text { de distribuição de } \\
\text { medicamentos }\end{array}$ \\
\hline & Aula 2 & $\begin{array}{l}\text { Como minimizar os riscos } \\
\text { de falhas na dispensação de } \\
\text { medicamentos? }\end{array}$ \\
\hline & Aula 3 & $\begin{array}{l}\text { Procedimentos para a } \\
\text { dispensação segura de } \\
\text { medicamentos }\end{array}$ \\
\hline \multirow{2}{*}{$\begin{array}{l}\text { Unidade 3: A } \\
\text { administração } \\
\text { segura de } \\
\text { medicamentos. }\end{array}$} & Aula 1 & $\begin{array}{l}\text { A administração segura de } \\
\text { medicamento e os nove } \\
\text { certos }\end{array}$ \\
\hline & Aula 2 & $\begin{array}{l}\text { Medidas adicionais } \\
\text { para a prática segura } \\
\text { de administração de } \\
\text { medicamento }\end{array}$ \\
\hline \multirow{2}{*}{$\begin{array}{l}\text { Unidade 4: } \\
\text { Notificação de } \\
\text { eventos adversos e } \\
\text { outros incidentes } \\
\text { relacionados a } \\
\text { medicamentos. }\end{array}$} & Aula 1 & O que é evento adverso? \\
\hline & Aula 2 & $\begin{array}{l}\text { Compreendendo o processo } \\
\text { de notificação de eventos } \\
\text { adversos }\end{array}$ \\
\hline
\end{tabular}

Fonte: Elaborado pelos autores. 
O MOOC foi disponibilizado na plataforma Moodle do Ambiente Virtual de Aprendizagem do Sistema Único de Saúde (Avasus), no dia 28 de fevereiro de 2018, por meio do seguinte domínio: https://avasus.ufrn.br/local/avasplugin/cursos/curso. php?id=222.

\section{População de estudo e amostra}

Do total de 7.135 participantes inscritos, 4.041 integralizaram o módulo, no período de março a dezembro de 2018. Foram incluídos no estudo 2.902 alunos, que concluíram o curso e assinaram o Termo de Consentimento Livre e Esclarecido (TCLE).

\section{Avaliação da satisfação e aprendizagem}

Aplicaram-se um questionário de satisfação após o curso e pré e pós-teste de conhecimento. O questionário de satisfação foi composto por seis perguntas, em que se utilizou a escala de Likert de cinco pontos ${ }^{11}$ : discordo totalmente; discordo parcialmente; neutro; concordo parcialmente; e concordo totalmente. Os testes de conhecimento foram criados a partir de um banco de dados de dez questões de múltipla escolha com o mesmo nível de dificuldade, julgadas por dois especialistas. $\mathrm{O}$ participante tinha a possibilidade de repetir os testes quantas vezes desejasse, porém a ordem das questões era aleatorizada a cada tentativa para minimizar o viés de memorização.

A aprendizagem foi mensurada por meio de duas variáveis:

- Melhoria absoluta: diferença entre a nota do pósteste e a do pré-teste (nota do pós-teste - nota do pré-teste).

- Melhoria relativa: percentual de melhoria em relação ao que era possível melhorar (média do pós-teste média do pré-teste)/(10 - média do pré-teste).

\section{ANÁLISE DOS DADOS}

Neste estudo, as variáveis dependentes foram a melhoria absoluta e a melhoria relativa; eas variáveis independentes, sexo, idade e categoria profissional. Para verificar a confiabilidade do questionário de satisfação, utilizou-se o alfa de Cronbach.

Para testar a associação entre as variáveis independentes coletadas (idade, sexo e categoria profissional) e as variáveis dependentes relativas à aprendizagem (melhoria absoluta e melhoria relativa), aplicaram-se análises bivariadas e multivariadas de regressão linear simples e múltipla, após confirmação da normalidade da variável dependente com teste de Kolgomorov-Smirnov. A significância adotada foi de $5 \%$, rejeitando hipóteses nulas de associação quando o $p$-valor foi menor que 0,05 . As análises estatísticas foram realizadas no programa SPSS, versão 22.0.

\section{Aspectos éticos}

Os alunos aceitaram participar da pesquisa assinando o TCLE inserido no ambiente virtual. O estudo foi aprovado pelo Comitê de Ética em Pesquisa (CEP) do Hospital Universitário Onofre Lopes (HUOL), sob Certificado de Apresentação de Apreciação Ética (CAAE) n 70587517.1.0000.5292.

\section{RESULTADOS}

Um total de 2.902 responderam aos instrumentos de avaliação. Houve predominância do público feminino $(72,6 \%)$, e a média de idade dos participantes foi de 34,2 anos $\pm 10,1$. Medicina foi a categoria mais prevalente entre os cursistas que informaram a formação, conforme a Tabela 1.

\section{Efeitos sobre o conhecimento dos participantes}

A partir da análise das notas dos testes, foi possível observar um aumento da média geral das pontuações obtidas pelos alunos $(p<0,05)$, verificado no teste T pareado. A média geral obtida no pós-teste $(8,74)$ foi $7 \%$ maior que a pontuação média do pré-teste $(8,10)$. A melhoria relativa foi de, aproximadamente, $32 \%$, o que demonstrou aprendizado significativo dos participantes, conforme mostra a Tabela 2. Farmácia foi a categoria profissional que apresentou melhor desempenho nos testes ( $p<0,05)$, com uma melhoria relativa de, aproximadamente, $43 \%$.

Tabela 1. Caracterização dos participantes avaliados

\begin{tabular}{cccc}
\hline \multicolumn{1}{c}{ Variáveis } & $\mathbf{n}$ & $\mathbf{f}$ \\
\hline Sexo & & & \\
& Feminino & 2.108 & $72,6 \%$ \\
Masculino & 778 & $26,8 \%$ \\
Não informado & 16 & $0,6 \%$ \\
\hline Idade & & \\
& $27-33$ & 733 & $25,3 \%$ \\
$34-40$ & 764 & $26,3 \%$ \\
$41>$ & 698 & $24,1 \%$ \\
Não informado & 684 & $23,6 \%$ \\
\hline Formação & 23 & $0,8 \%$ \\
Enfermagem & & \\
Farmácia & 134 & $4,6 \%$ \\
Medicina & 71 & $2,5 \%$ \\
Outras & 265 & $9,1 \%$ \\
Não informada & 24 & $0,8 \%$ \\
\hline Total & 2.408 & $83,0 \%$ \\
\hline
\end{tabular}

n: frequência absoluta; f: frequência relativa.

Fonte: Elaborada pelos autores. 
Tabela 2. Análise da performance dos cursistas nos testes pré e pós

\begin{tabular}{|c|c|c|c|c|c|c|c|c|}
\hline \multirow{2}{*}{ Variável } & \multirow{2}{*}{$\mathrm{n}$} & \multicolumn{2}{|c|}{ Pré-teste } & \multicolumn{2}{|c|}{ Pós-teste } & \multirow{2}{*}{$\begin{array}{l}\text { Melhoria } \\
\text { absoluta }\end{array}$} & \multirow{2}{*}{$\begin{array}{c}\text { Melhoria } \\
\text { relativa }\end{array}$} & \multirow{2}{*}{$p$ valor } \\
\hline & & Média & IC95\% & Média & IC95\% & & & \\
\hline \multicolumn{9}{|l|}{ Sexo } \\
\hline Feminino & 2.108 & 8,12 & $8,01-8,22$ & 8,74 & $8,66-8,83$ & 0,63 & $33 \%$ & $p<0,001$ \\
\hline Masculino & 778 & 8,08 & $7,90-8,26$ & 8,75 & $8,60-8,89$ & 0,67 & $35 \%$ & $p<0,001$ \\
\hline \multicolumn{9}{|c|}{ Categoria profissional } \\
\hline Enfermagem & 134 & 8,16 & $7,77-8,54$ & 8,66 & $8,30-9,01$ & 0,50 & $27 \%$ & $p<0,05$ \\
\hline Farmácia & 71 & 8,49 & $8,05-8,93$ & 9,13 & $8,81-9,46$ & 0,64 & $43 \%$ & $p<0,05$ \\
\hline Medicina & 265 & 8,72 & $8,45-8,99$ & 9,24 & $9,05-9,44$ & 0,52 & $41 \%$ & $\mathrm{p}<0,001$ \\
\hline Outras & 24 & 8,04 & $6,88-9,19$ & 9,17 & $8,63-9,70$ & 1,13 & $58 \%$ & $p=0,08$ \\
\hline \multicolumn{9}{|l|}{ Idade } \\
\hline$<26$ & 733 & 7,47 & $7,27-7,66$ & 8,22 & $8,05-8,38$ & 0,75 & $30 \%$ & $p<0,001$ \\
\hline $27-33$ & 764 & 8,03 & $7,85-8,20$ & 8,66 & $8,51-8,81$ & 0,64 & $32 \%$ & $\mathrm{p}<0,001$ \\
\hline $34-40$ & 698 & 8,36 & $8,18-8,54$ & 8,98 & $8,85-9,11$ & 0,62 & $38 \%$ & $p<0,001$ \\
\hline $41>$ & 684 & 8,63 & $8,46-8,80$ & 9,17 & $9,05-9,30$ & 0,54 & $40 \%$ & $\mathrm{p}<0,001$ \\
\hline
\end{tabular}

Melhoria absoluta $=$ média pós-teste - média pré-teste; melhoria relativa $=($ média pós-teste - média pré-teste $) /(10-$ média 1$)$.

Fonte: Elaborada pelos autores.

Tabela 3. Questionário de satisfação respondido por 2.902 cursistas avaliados no estudo

\begin{tabular}{|c|c|c|c|}
\hline Item & Concordância* & Neutro & Discordância** \\
\hline 1. Conteúdo do módulo foi satisfatório & $98,1 \%$ & $1,3 \%$ & $0,6 \%$ \\
\hline $\begin{array}{l}\text { 2. Conteúdo do módulo coerente com os objetivos de } \\
\text { aprendizagem }\end{array}$ & $97,6 \%$ & $1,9 \%$ & $0,4 \%$ \\
\hline 3. O módulo atendeu às suas expectativas & $94,6 \%$ & $4,2 \%$ & $1,2 \%$ \\
\hline $\begin{array}{l}\text { 4. As metodologias utilizadas facilitaram no processo de } \\
\text { aprendizagem }\end{array}$ & $93,9 \%$ & $5,0 \%$ & $1,1 \%$ \\
\hline $\begin{array}{l}\text { 5. O módulo propiciou a aquisição de novos conhecimentos e } \\
\text { habilidades }\end{array}$ & $95,6 \%$ & $3,8 \%$ & $0,6 \%$ \\
\hline $\begin{array}{l}\text { 6. Os novos conhecimentos e as habilidades adquiridos podem } \\
\text { ser aplicados na sua prática profissional }\end{array}$ & $96,7 \%$ & $2,6 \%$ & $0,7 \%$ \\
\hline Total & $96,1 \%$ & $3,1 \%$ & $0,8 \%$ \\
\hline
\end{tabular}

*Concordância: somatório de concordo totalmente e concordo parcialmente.

**Discordância: somatório de discordo totalmente e discordo parcialmente.

Fonte: Elaborada pelos autores.

Quanto à melhoria absoluta das notas, não foram identificadas associações significativas em relação às variáveis independentes medidas $(p>0,05)$. No entanto, considerando a melhoria relativa como variável dependente, após aplicação dos testes bivariados de associação, as variáveis com $p<0,02$ (idade e categoria profissional) foram incluídas no modelo inicial de regressão linear múltipla. Após o teste de regressão linear múltipla com o método Stepwise, identificou-se um modelo significativo $(p=0,008)$. A variável idade foi mantida no modelo, com beta de 1,36 (IC 95\% 0,35-2,36), revelando que, a cada aumento de um ano de idade, incrementa-se em 1,36 a estimativa da melhoria relativa.

\section{Satisfação dos participantes com a estratégia de ensino}

O questionário utilizado para avaliação de satisfação apresentou alfa de Cronbach de 0,83, o que demonstrou uma boa consistência interna do instrumento aplicado. $\mathrm{O}$ instrumento indicou elevada satisfação do público $(96,1 \%)$ em relação ao módulo educacional proposto para todos os itens avaliados (Tabela 3).

\section{DISCUSSÃO}

Este estudo contribui para a compreensão da importância da utilização de MOOC na disseminação em larga escala de conhecimento no ensino multiprofissional 
nos sistemas de saúde. A estratégia utilizada demonstrou boa aceitabilidade e melhoria significativa do conhecimento na temática de segurança no processo de medicação. Essa iniciativa pioneira se mostrou efetiva para o ensino inclusivo da segurança do paciente em um país continental que possui barreiras estruturais e geográficas consideráveis para o ensino presencial. Dessa forma, o módulo do Avasus disponibilizado se mostrou satisfatório e efetivo no ensino de segurança do paciente em nível de pós-graduação e no processo de educação permanente.

\section{Aceitabilidade do MOOC}

O nível de satisfação em relação à metodologia utilizada nomóduloeducacional propostoapresentou resultado bastante positivo com o questionário e corrobora outros estudos que apontam o uso de tecnologias da informação e comunicação (TICs) na facilitação da aprendizagem interprofissional ${ }^{12,13}$. A satisfação é fundamental para garantir a motivação do participante ao longo do curso, interferindo no rendimento do seu aprendizado e, consequentemente, na competência do profissional no seu respectivo cenário de prática ${ }^{14}$. Para se obter um processo significativo de aprendizagem, foi estabelecida uma linguagem direcionada ao participante, utilizando-se recursos como história em quadrinhos, infográficos e situações problemas, o que pode ter influenciado no grau de satisfação em relação à metodologia. A inserção de outros recursos que não foram utilizados no módulo avaliado, como videoaulas e o feedback, poderia preencher lacunas de expectativas verificadas no questionário de satisfação ${ }^{15}$.

\section{Aprendizagem promovida pelo MOOC}

A análise do nível de aprendizagem, por meio da aplicação de pré e pós-teste, demonstrou que o módulo educacional pode agregar novos conhecimentos, assim como outros estudos que utilizaram o mesmo método ${ }^{12,16}$. Entretanto, é preciso ter em mente que todo instrumento de avaliação é limitado, principalmente por se tratar de uma estratégia totalmente somativa ${ }^{17}$. Outro ponto a ser destacado é que não foi possível comparar diferenças do nível de aprendizagem na estratégia $\mathrm{EaD}$ com modelos presenciais, visto que não se identificaram cursos com a mesma temática abordada no módulo. Apesar disso, o módulo apresenta-se como uma estratégia alternativa para o ensino de segurança do paciente.

A elevada pontuação obtida no pré-teste pode estar relacionada ao grau de dificuldade do teste, porém observou-se correlação entre o número de tentativas e a maior pontuação. Acerca da diferença de rendimento entre participantes de Medicina, Enfermagem e Farmácia em cursos de EaD sobre aprendizagem interprofissional, já foi evidenciado que alunos de Farmácia e de Enfermagem apresentaram resultados superiores aos de Medicina ${ }^{18}$. $\mathrm{O}$ achado reforça a ideia de que o ensino de segurança do paciente ainda não faz parte dos objetivos de aprendizagem das escolas de saúde no Brasil. Dessa forma, faz-se necessária uma revisão dos currículos para contemplar o desenvolvimento da temática em uma abordagem interdisciplinar ${ }^{19}$.

Assim como outros estudos, a análise da performance por faixa etária indica que os alunos com idades superiores tendem a ter melhor desempenho nos cursos on-line ${ }^{20,21}$. Além do maior interesse dos alunos com maior faixa etária, a experiência profissional pode ser um fator determinante para a diferença de desempenho demonstrada ${ }^{21}$.

\section{Desafios para uso do MOOC na segurança do paciente}

A EaD apresenta desafios, como a resistência das pessoas em utilizar a estratégia de ensino desacreditando na eficácia da aprendizagem, por não haver um professor ou tutor mediando o conhecimento. Outra dificuldade é a má qualidade das bandas de conexão nas diversas localidades, o que prejudica a velocidade de transmissão de imagens e vídeos. Por fim, também cabe destacar a tecnofobia, que acomete pessoas que têm medo de usar as tecnologias, o que se torna um empecilho no processo de aprendizagem. Nesse caso, é fundamental uma estratégia eficiente no sentido de quebrar resistências e motivar o público-alvo para uso do ambiente virtual de forma disciplinada e auto-organizada, pois, nessa modalidade, o aluno deve passar de uma atitude passiva para uma atitude ativa, ou seja, ele deve se interessar pelo processo de aprendizado e buscar o conhecimento necessário ${ }^{22}$.

A abordagem multiprofissional utilizada no conteúdo do curso é um ponto positivo e pode ser um diferencial em relação a cursos na modalidade presencial. O curso abordou temas relacionados à prescrição, dispensação e administração de medicamentos em um único módulo, possibilitando a aquisição de novos conhecimentos de maneira transversal a diferentes categorias profissionais envolvidas no processo de medicação.

Casos clínicos apresentados por meio de histórias em quadrinhos possibilitaram aos participantes reconhecer o papel da segurança do paciente em diferentes situações que envolvem o cuidado, bem como destacaram a importância da comunicação e da colaboração interprofissional para evitar erros relacionados à saúde. Dessa forma, pode-se dizer que o módulo educacional possibilitou a remoção das limitações geográficas enfrentadas em modalidades presenciais e, principalmente, a quebra de paradigmas, saindo de um modelo tradicional de formação e educação permanente para uma modalidade pautada na efetiva integração entre os processos desenvolvidos e os profissionais. 
A educação em AVA também possibilita redução de custos com despesas gerais institucionais e viagen s $^{23,24}$. Contudo, esse fator não pôde ser investigado no presente estudo.

Em relação às novas competências adquiridas, a maioria dos alunos reconheceu que os novos conhecimentos e as habilidades aprendidos podem ser aplicados nos seus respectivos cenários de prática. Porém, a aplicação na prática não é possível na modalidade a distância, e isso é uma limitação desse modelo educacional. Outro fator limitante para a aplicação das novas competências individuais sobre segurança do paciente é a cultura organizacional, ou seja, quando os profissionais percebem que, em seus postos de trabalho, não são estabelecidas ações e normas fundamentais para a promoção de um ambiente seguro ${ }^{25}$.

\section{Limitações do estudo}

Os resultados limitam-se à análise do conhecimento e da satisfação quanto ao curso, não sendo possível avaliar seu efeito sobre habilidades e mudanças de atitudes que podem repercutir na qualidade do cuidado em saúde. Outros estudos como este deveriam ser realizados para medir o impacto de MOOC sobre a redução de EAM.

\section{CONCLUSÕES}

O uso do AVA pode ser uma boa estratégia para o ensino de temas relacionados à segurança do paciente. A experiência com o MOOC aqui relatada demonstrou assertividade na escolha da metodologia interativa para abordagem da segurança no processo de medicação, confirmando sua efetividade como recurso educacional para o ensino de segurança do paciente em larga escala. A iniciativa de abordar os temas prescrição, dispensação e administração de medicamentos em um único módulo possibilitou a aquisição de novos conhecimentos de maneira transversal a diferentes categorias profissionais envolvidas no processo de medicação. Portanto, o MOOC configura-se como uma potente ferramenta para o ensino interprofissional, como foi demonstrado neste estudo, e poderia ser utilizado para ampliar o ensino de segurança do paciente na graduação, na pós-graduação e na educação continuada nas profissões de saúde.

\section{CONTRIBUIÇÃO DOS AUTORES}

Thiago de Lima Pessoa desenvolveu o curso, revisou os textos para o AVA e participou da análise dos dados e da redação do artigo. Marise Reis de Freitas orientou o projeto de mestrado e participou da análise dos dados e da redação e revisão do artigo. Paulo José de Medeiros e Zenewton André da Silva Gama participaram da redação do artigo e da revisão final.

\section{CONFLITO DE INTERESSES}

Declaramos não haver conflito de interesses neste estudo.

\section{FINANCIAMENTO}

Declaramos que não houve financiamento neste estudo.

\section{REFERÊNCIAS}

1. Donaldson LJ, Kelley ET, Dhingra-Kumar N, Kieny MP, Sheikh A. Medication without harm: WHO's Third Global Patient Safety Challenge. Lancet. 2017;389(10080):1680-1.

2. Aspden P, Wolcott J, Lyle Bootman J, Cronenwett L. Preventing medication errors: Quality Chasm Series. Healthteamworks. Biz. Washington, DC Committee on Identifying and Preventing Medication Errors, Board on Health Care Services, Institute of Medicine; 2007. 480 p.

3. Leape L, Bates D, Cullen D, Cooper J, Demonaco H, Gallivan T, et al. Systems analysis of adverse drug events. JAMA. 1995;274(1):33-43;

4. Kohn LT, Corrigan JM, Donaldson MS. To err is human. In: Committee on Quality of Health Care in America I of $M$, organizer. To err is human: building a safer health system. Washington (DC): National Academies Press (US); 2000.

5. Pagnamenta A, Rabito G, Arosio A, Perren A, Malacrida R, Barazzoni F, et al. Adverse event reporting in adult intensive care units and the impact of a multifaceted intervention on drug-related adverse events. Ann Intensive Care. 2012; 2(47):1-10.

6. Rozenfeld S, Giordani F, Coelho S. Adverse drug events in hospital: pilot study with trigger tool. Rev Saude Publica. 2013;47(6):1102-11.

7. Organização Mundial da Saúde. Guia curricular de segurança do paciente da Organização Mundial da Saúde: edição multiprofissional. Rio de Janeiro: Autografia; 2016. 270 p.

8. Brasil. Documento de referência para o Programa Nacional de Segurança do Paciente. Brasília: Ministério da Saúde; 2014. 40 p.

9. Sales PRS, Marin MJS, Silva Filho CR. Integração academia-serviço na formação de enfermeiros em um hospital de ensino. Trab Educ Saúde. 2015;13(3):675-93.

10. Oliveira RM, Figueiredo SV, Sampaio RL, Gondim MM. Estratégias para promover segurança do paciente: da identificação dos riscos às práticas baseadas em evidências. Esc Anna Nery Rev Enferm. 2014;18(1):122-9.

11. da Silva Júnior SD, Costa FJ. Measurement and verification scales: a comparative analysis between the Likert and Phrase Completion Scales. PMKT - Revista Brasileira de Pesquisa de Marketing, Opinião e Mídia. 2014;15:1-15.

12. Curran V, Reid A, Reis $P$, Doucet $S$, Price $S$, Alcock $L$, et al. The use of information and communications technologies in the delivery of interprofessional education: a review of evaluation outcome levels. J Interprof Care. 2015;29(6):541-50.

13. dos Santos SGF, Marques IR. Uso dos recursos de internet na enfermagem: uma revisão. Rev Bras Enferm. 2006;59(2):212-6.

14. Vieira KM, Kunkel FIR, Mallmann EM. Satisfação dos estudantes de educação a distância da UFSM: uma análise multifatorial. Revista Eletrônica em Gestão, Educação e Tecnologia Ambiental. 30 jul 2013;12(12):2617-32.

15. Pizi ECG, Sasso ABM, Cavalheiro MQ, Stape THS, Coelho COL, Galhano GÁP. A plataforma Moodle no treinamento de estudantes de Odontologia na avaliação de restaurações classe II em resina composta. Rev ABENO. 2017;17(1):45-54

16. Tobase L, Peres HHC, de Almeida DM, Tomazini EAS, Ramos MB, Polastri TF. Instructional design in the development of an online course on Basic Life Support. Rev Esc Enferm USP. 26 mar 2018;51(3288):1-8.

17. Santos $L$. A articulação entre a avaliação somativa e a formativa, na prática pedagógica: uma impossibilidade ou um desafio? Ensaio: Avaliação e Políticas Públicas em Educação. 2016;24(92):637-69. 
18. Aziz Z, Teck LC, Yen PY. The attitudes of medical, nursing and pharmacy students to inter-professional learning. Procedia Soc Behav Sci. 2011;29:639-45.

19. Bohomol E, Freitas MAO, Cunha ICKO. Patient safety teaching in undergraduate health programs: reflections on knowledge and practice. Interface (Botucatu). 2016;20(58):727-41.

20. Valasek T. Student persistence in web-based courses: identifying a profile for success. Educ Resour Inf Cent. 2001;143:1-17.

21. Schneider SP, Germann CG. Technical Communication Quarterly Technical communication on the web: a profile of learners and learning environments. Tech Commun Q. 1999;8(1):37-48.

22. Laranjeira K S, Viana HB, Landim A, Carraro EC, Prates EAR. Benefícios e desafios da modalidade ensino à distância (e- learning) nas coorporações. Obs Econ Latinoam. 2017;227:1-24.
23. Hanna E, Soren B, Telner D, MacNeill H, Lowe M, Reeves S. Flying blind: the experience of online interprofessional facilitation. J Interprof Care. 2013;27(4):298-304.

24. Moore DE, Green JS, Gallis HA. Achieving desired results and improved outcomes: integrating planning and assessment throughout learning activities. J Contin Educ Health Prof. 2009;29(1):1-15.

25. Walsh K. Reflections of health care professionals on e-learning resources for patient safety. Proc (Bayl Univ Med Cent). 2018;31(1):35-6. 